\title{
Rationalization of a nanoparticle-based nicotine nanovaccine as an effective next-generation nicotine vaccine: A focus on hapten localization
}

\author{
Zongmin Zhao ${ }^{a}$, Yun $\mathrm{Hu}^{\text {a }}$, Theresa Harmon ${ }^{\mathrm{b}}$, Paul Pentel ${ }^{\mathrm{b}}$, Marion Ehrich ${ }^{\mathrm{c}}$, \\ Chenming Zhang a, *
}

a Department of Biological Systems Engineering, Virginia Tech, Blacksburg, VA 24061, USA

${ }^{\mathrm{b}}$ Minneapolis Medical Research Foundation, Minneapolis, MN 55404, USA

c Department of Biomedical Sciences and Pathobiology, Virginia Tech, Blacksburg, VA 24061, USA

\section{A R T I C L E I N F O}

\section{Article history:}

Received 22 March 2017

Received in revised form

15 May 2017

Accepted 19 May 2017

Available online 19 May 2017

\section{Keywords:}

Nicotine vaccine

Lipid-polymeric hybrid nanoparticle

Anti-nicotine antibody

Hapten localization

Smoking cessation

\begin{abstract}
A B S T R A C T
A lipid-polymeric hybrid nanoparticle-based next-generation nicotine nanovaccine was rationalized in this study to combat nicotine addiction. A series of nanovaccines, which had nicotine-haptens localized on carrier protein (LPKN), nanoparticle surface (LPNK), or both (LPNKN), were designed to study the impact of hapten localization on their immunological efficacy. All three nanovaccines were efficiently taken up and processed by dendritic cells. LPNKN induced a significantly higher immunogenicity against nicotine and a significantly lower anti-carrier protein antibody level compared to LPKN and LPNK. Meanwhile, it was found that the anti-nicotine antibodies elicited by LPKN and LPNKN bind nicotine stronger than those elicited by LPKN, and LPNK and LPNKN resulted in a more balanced Th1-Th2 immunity than LPKN. Moreover, LPNKN exhibited the best ability to block nicotine from entering the brain of mice. Collectively, the results demonstrated that the immunological efficacy of the hybrid nanoparticle-based nicotine vaccine could be enhanced by modulating hapten localization, providing a promising strategy to combatting nicotine addiction.
\end{abstract}

๑) 2017 Elsevier Ltd. All rights reserved.

\section{Introduction}

Tobacco smoking is one of the most significant public health threats the world has ever faced; approximately, 6 millions of premature deaths are attributed to tobacco use each year in the world [1-3]. Despite the strong desires to quit smoking, the majority of unassisted smokers usually relapse within the first month, and only $3-5 \%$ of them remain abstinent after 6 months [4]. Even with the help of pharmacological interventions, including nicotine replacement therapy, varenicline, and bupropion, the long-term smoking cessation rate at one year is disappointingly low (10-25\%) [5-8].

Nicotine vaccine has shown to be an attractive approach for smoking cessation $[9,10]$. Promisingly, some conjugate nicotine vaccines were successful in inducing strong immunogenicity as well as achieving high pharmacokinetic efficacy in preclinical and

\footnotetext{
* Corresponding author. 210 Seitz Hall, Department of Biological Systems Engineering, Virginia Tech, Blacksburg, VA 24061, USA.
}

E-mail address: chzhang2@vt.edu (C. Zhang). early-stage clinical trials [11-14]. However, none of them have shown overall enhanced smoking cessation rate over placebo so far, mainly due to the highly-varying and insufficient antibody titers [15-17]. Although great efforts have been made to improve their immunogenicity by modulating multiple factors [13,18-23], conjugate nicotine vaccines bear some intrinsic shortfalls, such as fast degradation, low nicotine loading capacity, low bioavailability, and poor recognition and uptake by immune cells, largely limiting their immunological efficacy.

To circumvent these disadvantages of conjugate nicotine vaccines, in our previous work, we designed the next-generation nicotine nanovaccines using nanoparticles (NPs) as delivery vehicles for antigen presentation [24-26]. Particularly, lipid-polymeric hybrid nanoparticle (NP)-based nicotine nanovaccines were demonstrated to induce significantly higher immunogenicity over the conjugate vaccines and result in better pharmacokinetic efficacy in mice [26,27].

Nicotine hapten is such a small molecule that can only elicit an immune response when attached to a carrier, such as proteins or 
nanoparticles [9]. In addition, a stimulating/carrier protein is always a necessity in NP-based nicotine vaccine, as it will stimulate Thelper cell formation that is required for B cell maturation $[9,28]$. Meanwhile, conjugating protein antigen to the surface of NPs could promote its delivery and presentation [29,30]. In our previous nanovaccine design, hapten was conjugated to the surface of protein antigens [26]. As the localization of haptens on vaccine NPs may potentially affect the recognition of antigens by immune cells, in this current work, we rationalized the design of a hybrid NPbased nicotine vaccine by studying the impact of hapten localization on its immunogenicity and pharmacokinetic efficacy. As shown in Scheme 1, three nanovaccines, which have haptens localized only on the carrier protein (LPKN), only on NP surface (LPNK), or on both (LPNKN), were synthesized. The immunogenicity and pharmacokinetic efficacy of nanovaccines were tested in mice.

\section{Materials and methods}

\subsection{Materials}

Lactel $^{\circledR}(50: 50$ poly(lactic-co-glycolic acid) (PLGA)) was purchased from Durect Corporation (Cupertino, CA, USA). Keyhole limpet hemocyanin (KLH) was purchased from Stellar Biotechnologies (Port Hueneme, CA, USA). Alexa Fluor ${ }^{\circledR} 647$ NHS ester (AF647), Alexa Fluor ${ }^{\circledR}$ 350 NHS ester (AF350), 1-Ethyl-3-[3-dimethylaminopropyl] carbodiimide hydrochloride (EDC), and N-hydroxysulfosuccinimide (SulfoNHS) were purchased from Thermo Fisher Scientific (Rockford, IL, USA). 1,2-Dioleoyl-3-trimethylammonium-propane (DOTAP), cholesterol (CHOL), 1,2-diphytanoyl-sn-glycero-3-phosphoethanolamine-N(7-nitro-2-1,3-benzoxadiazol-4-yl) (ammonium salt) (NBD-PE), 1,2distearoyl-sn-glycero-3-phosphoethanolamine-N-[maleimide(polyethylene glycol)-2000] (ammonium salt) (DSPE-PEG2000-maleimide), and 1,2-distearoyl-sn-glycero-3-phosphoethanolamine-N-[amino(polyethylene glycol)-2000] (ammonium salt) (DSPE-PEG2000amine) were purchased from Avanti Polar Lipids (Alabaster, AL, USA). O-Succinyl-3'-hydroxymethyl-( \pm )-nicotine (Nic) was purchased from Toronto Research Chemicals (North York, ON, Canada). All other chemicals were of analytical grade.

\subsection{Fabrication of PLGA NPs by nanoprecipitation}

PLGA NPs were fabricated by a nanoprecipitation method [31]. In brief, $20 \mathrm{mg}$ of PLGA was dissolved in $2 \mathrm{~mL}$ of acetone. The PLGAin-acetone organic solution was injected into $10 \mathrm{~mL}$ of $0.5 \%$ PVA aqueous phase by a vertically mounted syringe pump with magnetic stir agitation (1200 rpm). The resultant suspension was placed under vacuum for $6 \mathrm{~h}$ to eliminate the organic solvent. PLGA NPs were collected by centrifugation at $10,000 \mathrm{~g}, 4^{\circ} \mathrm{C}$ for $30 \mathrm{~min}$.

\subsection{Fabrication of lipid-polymeric hybrid NPS}

Lipid-polymeric hybrid NPs were fabricated with a previously reported hydration-sonication method [26,32]. In brief, $2.5 \mathrm{mg}$ of lipid mixture consisting of different molar ratios of DOTAP, DSPEPEG2000-maleimide, DSPE-PEG2000-amine, and CHOL, was evaporated to form a lipid film. The lipid film was hydrated with $0.01 \mathrm{M}$ phosphate buffer saline (PBS) and sonicated for $2 \mathrm{~min}$ to form a liposome suspension. Lipid-polymeric hybrid NPs were assembled by coating liposomes to PLGA NPs (PLGA: lipids $=10: 1$ $(\mathrm{w} / \mathrm{w}))$ via sonication for $10 \mathrm{~min}$. Lipid-polymeric hybrid NPs were collected by centrifugation at $10,000 \mathrm{~g}, 4{ }^{\circ} \mathrm{C}$ for $30 \mathrm{~min}$. The PLGA cores were labeled by Nile Red, and the number of NPs per mg was estimated by flow cytometry using an Amnis ImageStream ${ }^{\mathrm{X}}$ Mark 2 imaging flow cytometer.

\subsection{Synthesis of Nic-KLH conjugates}

Nic-KLH conjugates were synthesized by an EDC/NHS-mediated reaction as reported previously [26]. Specifically, the Nic-KLH conjugates used for preparing LPKN or LPNKN nanovaccines were synthesized by reacting $1.2 \mathrm{mg}$ or $2.4 \mathrm{mg}$ of Nic hapten with $5 \mathrm{mg}$ of $\mathrm{KLH}$. Hapten densities of Nic-KLH conjugates were estimated by a 2,4,6-trinitrobenzene sulfonic acid-based method as reported previously [33]. Nic-BSA conjugate was synthesized using the same method.

\subsection{Preparation of nanovaccine NPS}

LPKN nanovaccine NPs were assembled with the method reported previously [26]. In brief, an appropriate amount of Traut's reagent was added into the Nic-KLH conjugates equivalent to $2 \mathrm{mg}$ of KLH in $0.5 \mathrm{~mL}$ of PBS and reacted for $1 \mathrm{~h}$ to form thiolated NicKLH. The thiolated Nic-KLH equivalent to $1 \mathrm{mg}$ of KLH was conjugated to $30 \mathrm{mg}$ of lipid-polymeric hybrid NPs by reacting the thiolated Nic-KLH with maleimide groups in the lipid layer of NPs for $2 \mathrm{~h}$. Unconjugated Nic-KLH was separated by centrifugation at $10,000 \mathrm{~g}, 4^{\circ} \mathrm{C}$ for $30 \mathrm{~min}$, and quantified by the bicinchoninic acid assay. Negative control was prepared following a similar procedure, except that KLH, instead of Nic-KLH, was conjugated to NP surface.

For LPNK and LPNKN synthesis, Nic-haptens were conjugated to
LPKN

(haptens only on carrier protein)
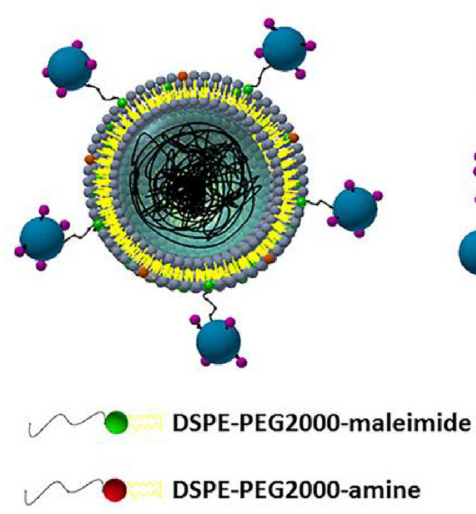

LPNK

(haptens only on nanoparticle)

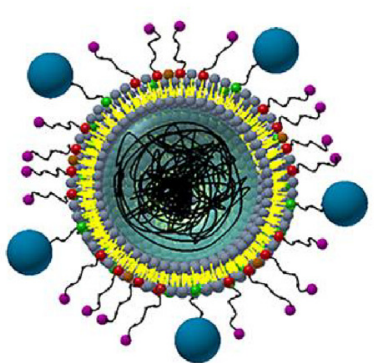

Nicotine hapten

DOTAP

Cholesterol
LPNKN

(haptens on both carrier protein and nanoparticle)

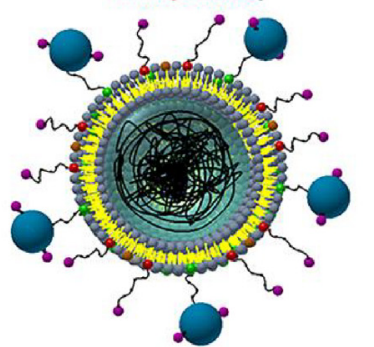

KLH

PLGA nanoparticle

Scheme 1. Schematic illustration of the structure of hybrid NP-based nicotine nanovaccines with different hapten localizations. 
the surfaces of hybrid NPs via an EDC/NHS-mediated reaction. In brief, an aliquot of Nic-haptens was activated for $30 \mathrm{~min}$ in $0.3 \mathrm{~mL}$ of activation buffer ( $0.1 \mathrm{M}$ MES, $0.5 \mathrm{M} \mathrm{NaCl}, \mathrm{pH}$ 6.0) by adding EDC and NHS (Nic: EDC: NHS = 1:10:10). Nic-hapten-conjugated hybrid NPs (LPN) were synthesized by reacting the activated Nic-haptens with $30 \mathrm{mg}$ of hybrid NPs in $2 \mathrm{~mL}$ of coupling buffer (0.1 M sodium phosphate, $0.15 \mathrm{M} \mathrm{NaCl}$, pH 7.2) for $10 \mathrm{~h}$. Unconjugated Nichaptens were eliminated by dialysis and quantified by HPLC using a Luna C18 (2) reverse phased chromatography column and a UV detector (at $254 \mathrm{~nm}$ ). LPNK and LPNKN NPs were assembled by conjugating KLH or Nic-KLH to LPN NPs with the same method as LPKN nanovaccine. Nanovaccine NPs were collected by centrifugation at $10,000 \mathrm{~g}, 4^{\circ} \mathrm{C}$ for $30 \mathrm{~min}$, and stored at $2{ }^{\circ} \mathrm{C}$ for later use.

\subsection{Characterization of NPS}

Size distribution and zeta potential of NPs were measured in $0.01 \mathrm{M} \mathrm{pH} 7.4$ PBS on a Nano ZS Zetasizer (Malvern Instruments, Worcestershire, United Kingdom) at $25^{\circ} \mathrm{C}$. The morphology of NPs was characterized by transmission electron microscopy (TEM) on a JEM 1400 transmission electron microscope (JEOL, Tokyo, Japan). Fluorescent nanovaccine NPs, in which the lipid layer was labeled by NBD, and AF647 and AF350 were conjugated to KLH and NP surface, respectively, were imaged on a Zeiss LSM 510 laser scanning microscope (Carl Zeiss, Oberkochen, Germany). The Fourier transform infrared (FT-IR) spectra of NPs were recorded on a Thermo Nicolet 6700 FT-IR spectrometer (Thermo Fisher Scientific, Waltham, MA).

\subsection{Cellular uptake of nanovaccine NPs in dendritic cells (DCs)}

The uptake of nanovaccine NPs by DCs was studied by flow cytometry assay (FCA). NBD-labeled LPKN, LPNK, and LPNKN NPs were prepared by adding $2.5 \%$ of NBD into lipid mixture. JAWSII (ATCC $^{\circledR}$ CRL-11904TM) immature DCs $\left(2 \times 10^{6} /\right.$ well $)$ were seeded into 24-well plates and cultured overnight. Cells were treated with $20 \mu \mathrm{g}$ of NBD-labeled nanovaccine NPs for $15 \mathrm{~min}$ or $2 \mathrm{~h}$. After washed 3 times with PBS, cells were detached from the culture plates using trypsin/EDTA solution and collected by centrifugation at $200 \mathrm{~g}$ for $10 \mathrm{~min}$. Cell pellets were re-suspended in PBS. Samples were immediately analyzed on a flow cytometer (FACSAria I, BD Biosciences, Franklin Lakes, NJ, USA).

The cellular uptake and processing of nanovaccine NPs were analyzed by confocal laser scanning microscopy (CLSM). AF647- and NBD-labeled NPs were prepared according to the method described above, except that AF647-KLH was conjugated to NPs and $2.5 \%$ of NBD was added into lipids for labeling. Cells $\left(2 \times 10^{5} /\right.$ chamber $)$ were seeded into 2-well chamber slides and cultured overnight. Cells were treated with $20 \mu \mathrm{g}$ of AF647- and NBD-labeled nanovaccine NPs for 15 min or $2 \mathrm{~h}$. Cells were then washed using PBS and fixed with freshly prepared $4 \%(\mathrm{w} / \mathrm{v})$ paraformaldehyde for $10 \mathrm{~min}$. The membrane of cells was permeabilized by adding $0.5 \mathrm{~mL}$ of $0.1 \%(\mathrm{v} / \mathrm{v})$ Triton $^{\mathrm{TM}} \mathrm{X}-100$ for $10 \mathrm{~min}$. Cell nuclei were stained by $4^{\prime}, 6-$ diamidino-2-phenylindole (DAPI). The intracellular distribution of NPs was visualized on a Zeiss LSM 510 laser scanning microscope.

\subsection{Immunization of mice with nicotine nanovaccines}

All animal studies were carried out following the National Institutes of Health guidelines for animal care and use. Animal protocols were approved by the Institutional Animal Care and Use Committee at Virginia Tech. Female Balb/c mice (6-7 weeks of age, $16-20 \mathrm{~g}, 6$ per group) were immunized subcutaneously with a total volume of $200 \mu \mathrm{L}$ of nicotine vaccines containing $25 \mu \mathrm{g}$ of $\mathrm{KLH}$ antigen on days 0,14 , and 28 . The subcutaneous injection site was over the shoulder of mice (into the loose skin over the neck). For the negative control group, mice were immunized with KLH associated hybrid NPs without Nic-hapten conjugation containing $25 \mu \mathrm{g}$ of KLH. For the blank group, mice were injected with $200 \mu \mathrm{L}$ of sterilized PBS. Blood was collected from the retro-orbital plexus under isoflurane anesthesia on days $0,12,26$, and 40 .

\subsection{Measurement of titers of anti-nicotine IgG antibody, anti- nicotine IgG subclass antibody, and anti-KLH antibody}

Anti-nicotine IgG and IgG-subclass antibody titers were measured by an enzyme-linked immunosorbent assay (ELISA) according to a previously reported method [25]. Anti-KLH antibody titers were measured following a similar protocol, except that KLH was used as a coating material. Antibody titer was defined as the dilution factor at which absorbance at $450 \mathrm{~nm}$ declined to half maximal.

\subsection{Measurement of anti-nicotine antibody affinity}

The relative affinity of anti-nicotine antibody induced by nicotine nanovaccines was measured by a competition ELISA method [34]. In brief, serum samples were diluted to achieve absorbance values of around 1.0 at $450 \mathrm{~nm}$. Nicotine was serially diluted from $10^{-2} \mathrm{M}$ to $10^{-7} \mathrm{M}$. One hundred $\mu \mathrm{L}$ of nicotine solutions were added into Nic-BSA coated plates, and $100 \mu \mathrm{L}$ of serum samples were subsequently added to plates. The other steps were the same as in measuring anti-nicotine antibody titers. Percent inhibition was calculated at each nicotine concentration and plotted against log nicotine concentration. The concentration at which 50\% inhibition was achieved $\left(\mathrm{IC}_{50}\right)$ was extrapolated for each sample.

\subsection{Pharmacokinetic study in mice}

The pharmacokinetic study was conducted using a method reported previously [26]. In brief, mice were administered $0.06 \mathrm{mg} / \mathrm{kg}$ nicotine subcutaneously two weeks after the second booster immunization (on day 42). Brain and serum samples were collected 3 min post nicotine dosing. Nicotine concentration in the brain and serum was measured by GC/MS as reported previously [35].

\subsection{Histopathological analysis}

Histopathological analysis was conducted to detect lesions of mouse organs caused by the immunization with nicotine vaccines following a method reported previously [26]. On day 42, organs of immunized mice, including heart, kidney, liver, lung, and spleen, were harvested and fixed in $10 \%$ buffered formalin. Tissue blocks were stained with hematoxylin and eosin $(\mathrm{H} \& \mathrm{E})$ according to the method described before [25] and imaged on a Nikon Eclipse E600 light microscope.

\subsection{Statistical analyses}

Data are expressed as means \pm standard deviation unless specified. Comparisons among multiple groups were conducted using one-way ANOVA followed by Tukey's HSD test. Differences were considered significant when the p-values were less than 0.05 .

\section{Results}

\subsection{Verification of Nic-hapten conjugate chemistry}

CLSM was employed to verify the Nic-hapten conjugate chemistry. AF350-NHS and AF647-NHS, which have the same reactive groups as the Nic-hapten, were used to simulate the hapten and conjugated to NP surface and KLH, respectively. Hybrid NPs were 
labeled by NBD. The co-localization of AF647 with NBD suggested the successful conjugation of the model hapten to KLH (the upper panel of Fig. 1A), and thus verified the conjugate chemistry for LPKN synthesis. On the other hand, the overlapping of AF350, AF647, and NBD indicated the efficient conjugation of the model haptens to NP surface and the successful association of the model hapten-KLH conjugate to NPs (the lower panel of Fig. 1A), and verified the conjugate chemistry for LPNK and LPNKN synthesis.

FT-IR was used to further validate the conjugate chemistry for nanovaccine synthesis. Specific peaks of both Nic-hapten (636 and $\left.708 \mathrm{~cm}^{-1}\right)$ and $\mathrm{KLH}\left(1654 \mathrm{~cm}^{-1}\right)$ are evident in the spectrum of NicKLH conjugate (Fig. 1B), suggesting the efficient conjugation of Nichapten to KLH. Similarly, characteristic peaks of Nic-hapten (858 and $949 \mathrm{~cm}^{-1}$ ) appeared in the spectrum of LPN NPs (Fig. 1C), revealing the successful attachment of Nic-hapten to NP surface. Finally, the spectra of all three nanovaccines included characteristic peaks of both Nic-hapten and KLH/Nic-KLH (Fig. 1D), indicating the successful synthesis of nanovaccines.

\subsection{Characterization of nanovaccine NPS}

Nanovaccine NPs were characterized morphologically using TEM (Fig. 2A). A core-shell structure was clearly shown on hybrid NPs, which was displayed as a bright core and dark shell. All three nanovaccine NPs had similar morphological features. Specifically, multiple black dots that were KLH/Nic-KLH showed on the surface of NPs, again suggesting the successful conjugation of protein antigens to hybrid NP surface. The conjugation efficiency of Nic-KLH/KLH was
$82.3 \pm 5.4 \%, 85.3 \pm 7.4 \%$, and $80.2 \pm 6.7 \%$, for LPKN, LPNK, and LPNKN, respectively (Table 1). The Nic-hapten densities of LPKN, LPNK, and LPNKN were $(6.32 \pm 0.39) \times 10^{4} / \mathrm{NP},(5.89 \pm 0.67) \times 10^{4} / \mathrm{NP}$, and $(6.02 \pm 0.53) \times 10^{4} / \mathrm{NP}$, respectively (Table 1$)$, suggesting that the three nanovaccines with different hapten localizations had similar overall hapten densities.

Moreover, the physicochemical properties of NPs were characterized. The three nanovaccines exhibited a similar average diameter, which was 118.1, 122.8, and $115.7 \mathrm{~nm}$ for LPKN, LPNK, and LPNKN nanovaccines, respectively (Table 1 ). Dynamic light scattering data revealed that all three nanovaccines had a similar size distribution, with most particles being smaller than $200 \mathrm{~nm}$ (Fig. 2B). The zetapotentials were $5.46 \pm 0.25,2.85 \pm 0.23$, and $4.69 \pm 0.24 \mathrm{mV}$, for LPKN, LPNK, and LPNKN, respectively (Table 1), revealing that all three nanovaccines were positively charged at $\mathrm{pH}$ 7.4.

The stability of nanovaccines, indicated by size change, was tested in PBS and DI water for 7 weeks, a period of time that is sufficient for the entire vaccination regimen and a short period of storage. The size change of all three nanovaccines was less than $20 \mathrm{~nm}$ in PBS over the entire study period (Fig. 2C), suggesting the nanovaccines were highly stable in PBS for at least 7 weeks. The nanovaccines appeared to be less stable in water. However, the size change of nanovaccines was still less than $30 \mathrm{~nm}$ for 7 weeks in DI water (Fig. 2D).

\subsection{Cellular uptake of nanovaccine NPS}

The cellular uptake of nanovaccine NPs was studied in DCs by FCA. The uptake of nanovaccines displayed a time-dependent

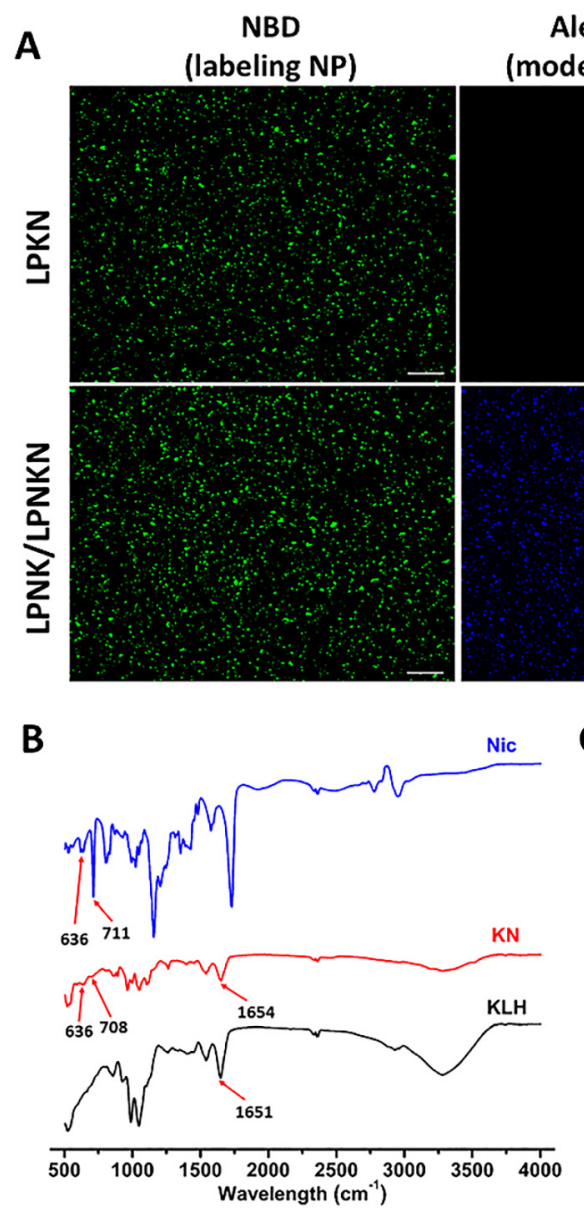

Alexa Fluor 350 (model hapten on NP)

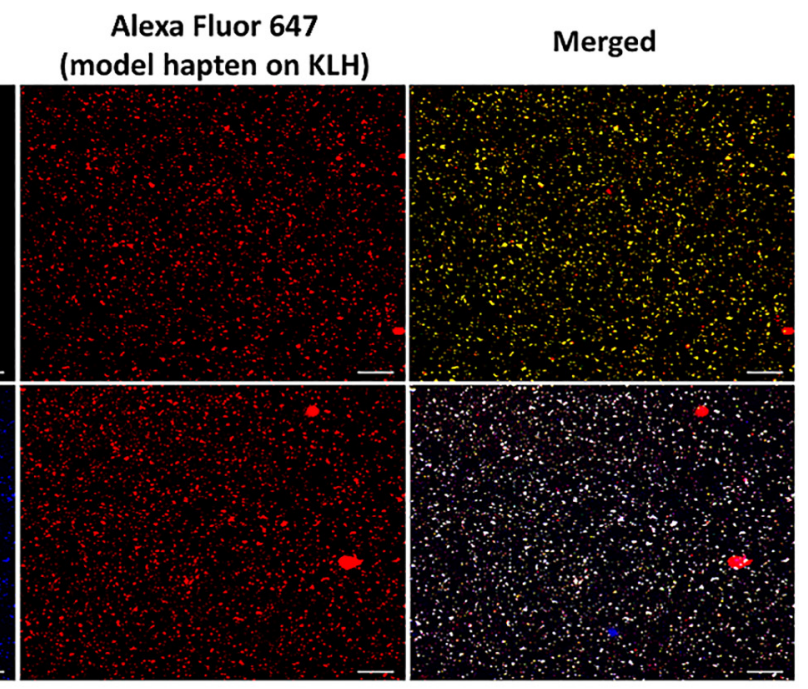

C
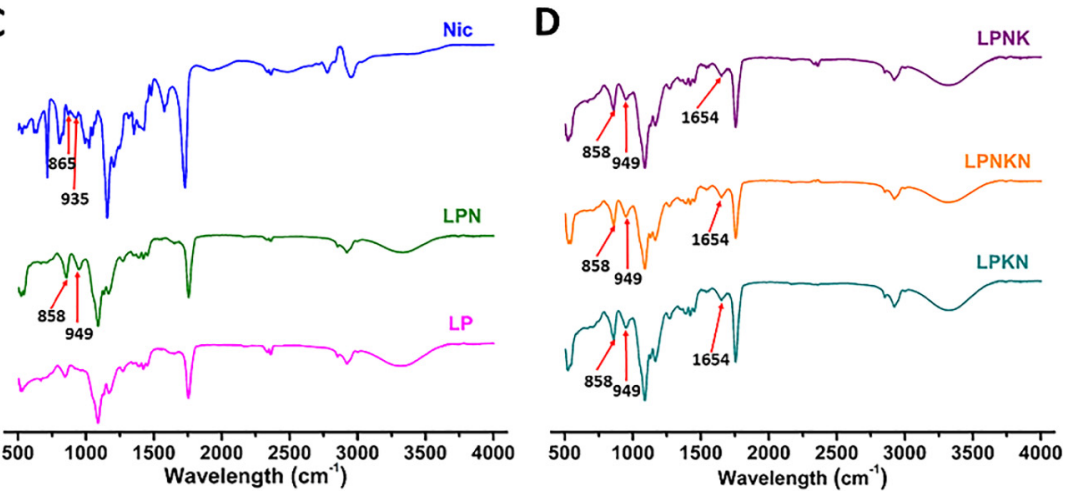

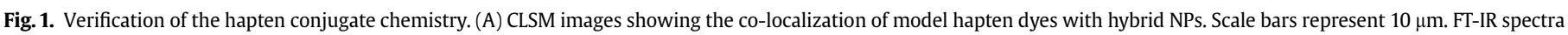
of Nic-hapten (Nic), Nic-KLH conjugate (KN), and KLH (B); Nic-hapten, hybrid NPs (LP), and Nic-hapten-conjugated LPN NPs (LPN) (C); LPKN, LPNK, and LPNKN (D). 
A

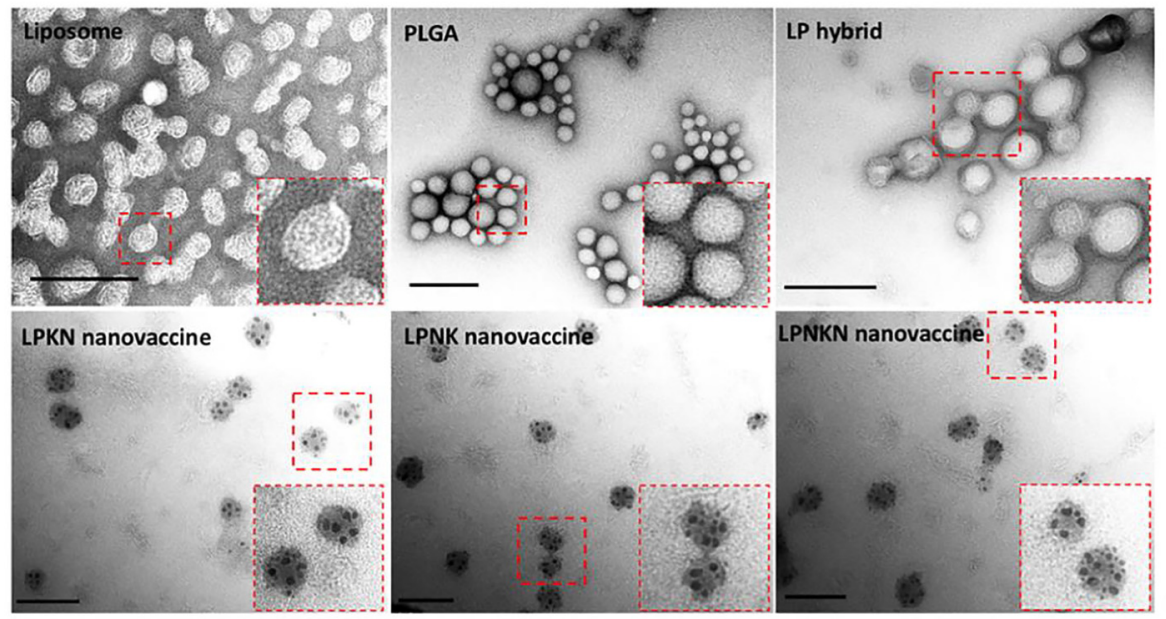

B

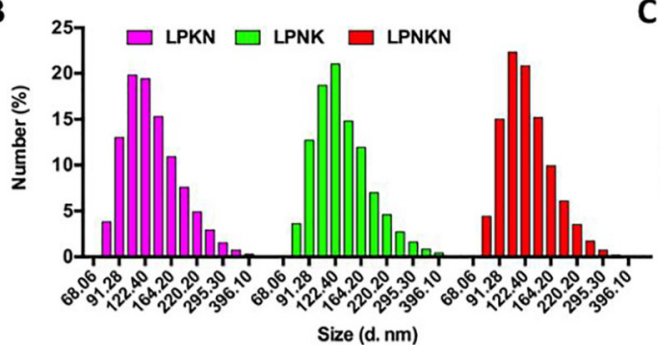

C

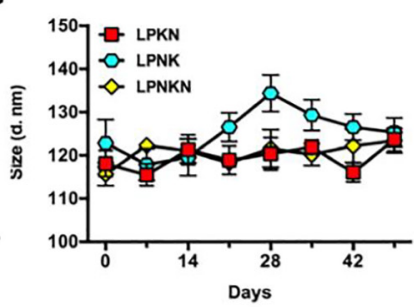

D

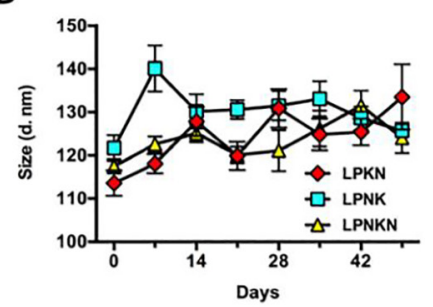

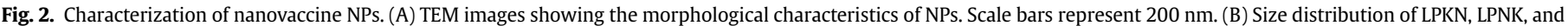
LPNKN NPs. (C) and (D) show the stability of nanovaccines in PBS and DI water at $4{ }^{\circ} \mathrm{C}$, respectively.

manner. After 15 min's incubation, except for LPKN, only small portions of cells had taken up NPs (Fig. 3A and B). The percentages of NBD-positive cells were $43.0 \pm 8.3 \%, 19.2 \pm 1.76 \%$, and $24.5 \pm 0.8 \%$ for LPKN, LPNK, and LPNKN, respectively (Fig. 3B). The corresponding median NBD intensity was $773 \pm 52,522 \pm 30$, and $540 \pm 6$, respectively (Fig. 3C). After 120 min's incubation, more NPs were internalized for all three nanovaccines. Particularly, the percentages of NBD-positive cells were $93.0 \pm 1.4 \%, 77.3 \pm 0.9 \%$, and $84.3 \pm 3.0 \%$, for LPKN, LPNK, and LPNKN, respectively (Fig. 3B); and the median NBD intensity was $1560 \pm 44,1217 \pm 28$, and $1237 \pm 34$, respectively (Fig. 3C). The data of both NBD-positive cells and NBD median intensity revealed that LPKN were taken up by dendritic cells more rapidly than LPNK and LPNKN.

The uptake and processing of nanovaccines were further studied by CLSM. Consistent with the FCA data, the uptake of nanovaccine NPs was time-dependent (Fig. 4). After 15 min's incubation, weak NBD and AF647 fluorescences were shown in cells. This suggested cells had taken up small amounts of nanovaccine NPs within 15 min. In contrast, the fluorescence of NBD and AF647 was very strong in cells after 120 min's incubation, indicating more NPs were taken up with time. Interestingly, the processing of nanovaccines appeared to be step-wise in cells. After $15 \mathrm{~min}$, NBD fluorescence was widely distributed in cells while AF647 fluorescence was displayed through individual particles. This phenomenon indicates that the lipid-layer was removed from the hybrid NPs to release protein antigens, but the protein antigens had not been efficiently processed. After $120 \mathrm{~min}$, both NBD and AF647 fluorescence were widely distributed in cells, revealing that protein antigens had been effectively processed to small peptides. Moreover, consistent with the FCA data, LPKN was observed to be more efficiently taken up by dendritic cells than LPNK and LPNKN, as both NBD and AF647 fluorescence were stronger in the LPKN group, especially at $120 \mathrm{~min}$.

\subsection{Immunogenicity of nanovaccines against nicotine and the carrier protein}

The immunogenicity of nanovaccines against nicotine was evaluated in mice, and the results are shown in Fig. 5A. No anti-nicotine antibody titers were detected in the negative control group on all days in which mice were immunized with KLH associated hybrid NPs without conjugated hapten. After the primary immunization, the anti-nicotine antibody titers of LPKN, LPNK, and LPNKN on day 12 were $(1.3 \pm 0.1) \times 10^{3},(1.6 \pm 0.2) \times 10^{3}$, and $(2.3 \pm 0.3) \times 10^{3}$, respectively. After the first booster immunization, anti-nicotine antibody titers on day 26 significantly increased over that on day 12. The titers were $(9.2 \pm 2.2) \times 10^{3},(9.8 \pm 6.0) \times 10^{3}$, and $(21.9 \pm 4.5) \times 10^{3}$ for LPKN, LPNK, and LPNKN, respectively. After the second booster immunization, anti-nicotine antibody titers considerably increased again on day 40 , which were $(15.5 \pm 2.3) \times 10^{3}$,

Table 1

Characteristics of nanovaccines with different hapten localizations.

\begin{tabular}{|c|c|c|c|c|c|}
\hline Nanovaccines & Size (d. nm) & Zeta-potential (mV) & PDI & $\begin{array}{l}\text { KLH/Nic-KLH conjugation } \\
\text { efficiency (\%) }\end{array}$ & $\begin{array}{l}\text { Nic-hapten density } \\
\left(\# / \times 10^{4} / \mathrm{NP}\right)\end{array}$ \\
\hline LPKN & $118.1 \pm 3.0$ & $5.46 \pm 0.25$ & $0.11 \pm 0.02$ & $82.3 \pm 5.4$ & $6.32 \pm 0.39$ \\
\hline LPNK & $122.8 \pm 5.5$ & $2.85 \pm 0.23$ & $0.15 \pm 0.03$ & $85.3 \pm 7.4$ & $5.89 \pm 0.67$ \\
\hline LPNKN & $115.7 \pm 2.7$ & $4.69 \pm 0.24$ & $0.14 \pm 0.03$ & $80.2 \pm 6.7$ & $6.02 \pm 0.53$ \\
\hline
\end{tabular}


A
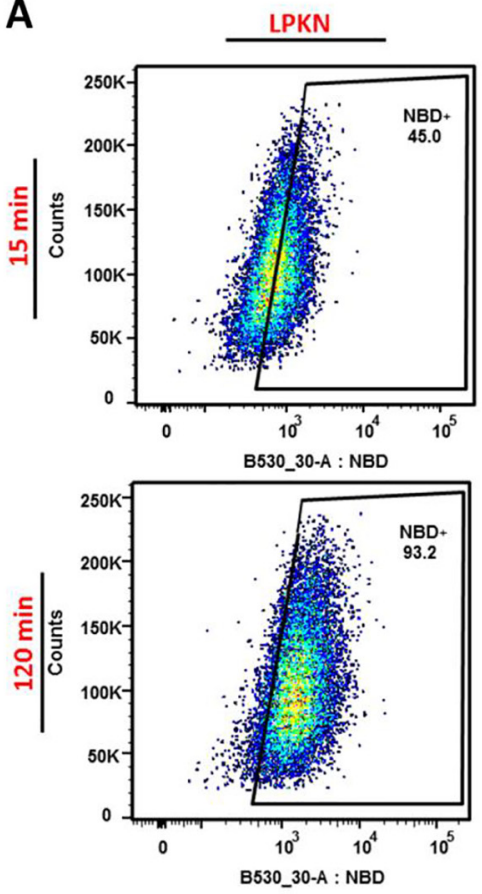
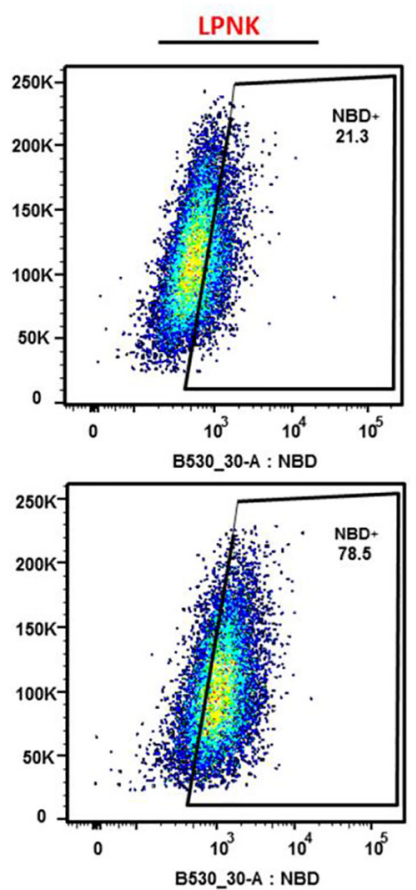

LPNKN
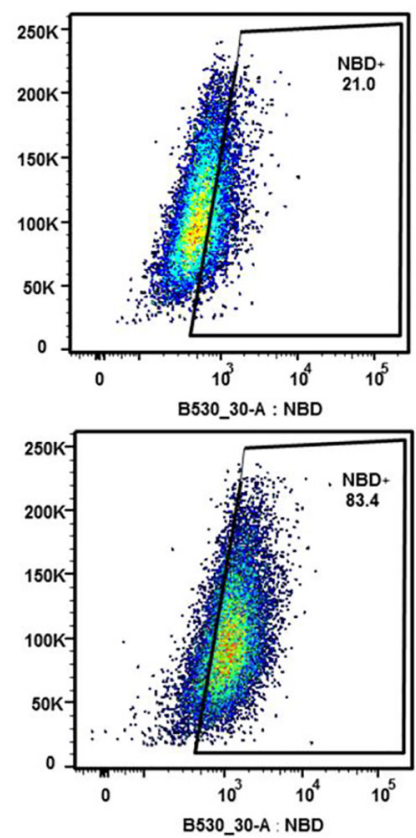

B

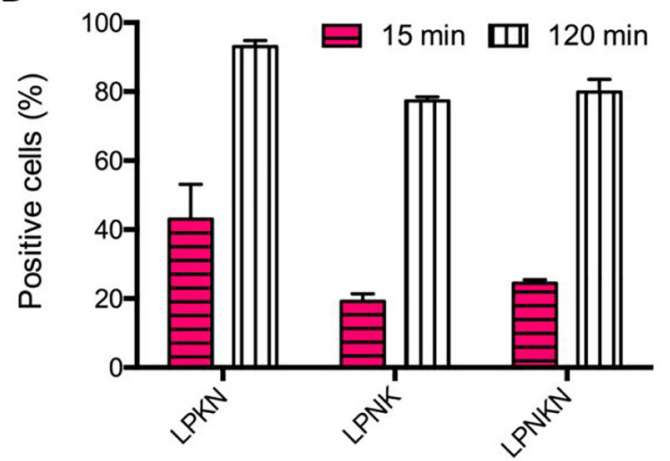

C

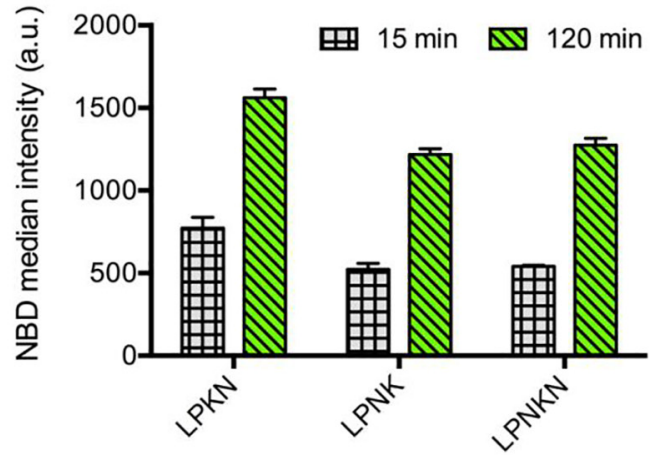

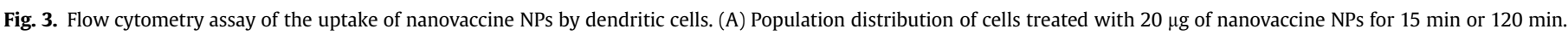
The percentage of NBD-positive cells (B) and NBD median intensity in cells (C) were analyzed.

$(13.1 \pm 4.1) \times 10^{3}$, and $(31.0 \pm 12.4) \times 10^{3}$ for LPKN, LPNK, and LPNKN, respectively. Statistical analysis suggested that LPNKN generated significantly higher anti-nicotine antibody titers than LPKN and LPNK $(\mathrm{p}<0.05)$, while LPKN and LPNK induced comparable titers $(\mathrm{p}>0.95)$, on all studied days.

Titers of anti-KLH antibody were also monitored. The results are shown in Fig. 5B. Similar to anti-nicotine antibody titers, anti-KLH antibody titers significantly increased after each immunization. On all studied days, the negative control induced the highest level of anti-KLH antibody. For the nanovaccines with different hapten localizations, the anti-KLH antibody titers were in the order of LPKN $>$ LPNK $>$ LPNKN for all studied days. The differences among different nanovaccine groups were significant $(p<0.05)$ on days 26 and 40, except for LPKN and LPNK. Specifically, end-point titers of $(79.1 \pm 14.1) \times 10^{3},(47.9 \pm 4.3) \times 10^{3},(44.7 \pm 7.1) \times 10^{3}$, and $(21.8 \pm 2.6) \times 10^{3}$ were detected in the negative control, LPKN, LPNK, and LPNKN groups, respectively.

\subsection{Affinity of anti-nicotine antibody induced by nanovaccines}

The binding affinity of nicotine to anti-nicotine antibodies elicited by nanovaccines was estimated by competition ELISA, and the time-course antibody affinity on days 12,26 , and 40 was shown in Fig. 6A. On day $12, \mathrm{IC}_{50}$ for LPKN, LPNK, and LPNKN was $1085 \pm 1103,1380 \pm 460$, and $1077 \pm 319 \mu \mathrm{M}$, respectively. On day 26 , IC 50 decreased to be $29 \pm 19,468 \pm 302$, and $29 \pm 31 \mu \mathrm{M}$, for LPKN, LPNK, and LPNKN, respectively. Evidently, the first booster immunization significantly promoted the maturation of antibodies. Interestingly, after the second booster immunization (on day 40), the affinity of antibodies induced by the three nanovaccines decreased. The IC 50 was $115 \pm 162,1004 \pm 1276$, and $132 \pm 51 \mu \mathrm{M}$ for LPKN, LPNK, and LPNKN, respectively. The affinity of antibodies induced by LPKN and LPNKN was considerably higher over LPNK on all the studied days. Specially, statistical comparison suggested that the end-point affinity of antibodies elicited by LPKN and LPNKN was significantly higher than that induced by LPNK, and no significant differences existed between LPKN and LPNKN (Fig. 6B).

\subsection{IgG subclass distribution of anti-nicotine antibodies}

The subtype distribution of anti-nicotine IgG antibodies induced by the nanovaccines on day 40 was analyzed. As shown in Fig. 7A-D, IgG1 was the dominant one among all four subtypes for all three nanovaccines. In concordance with the total IgG titers, 

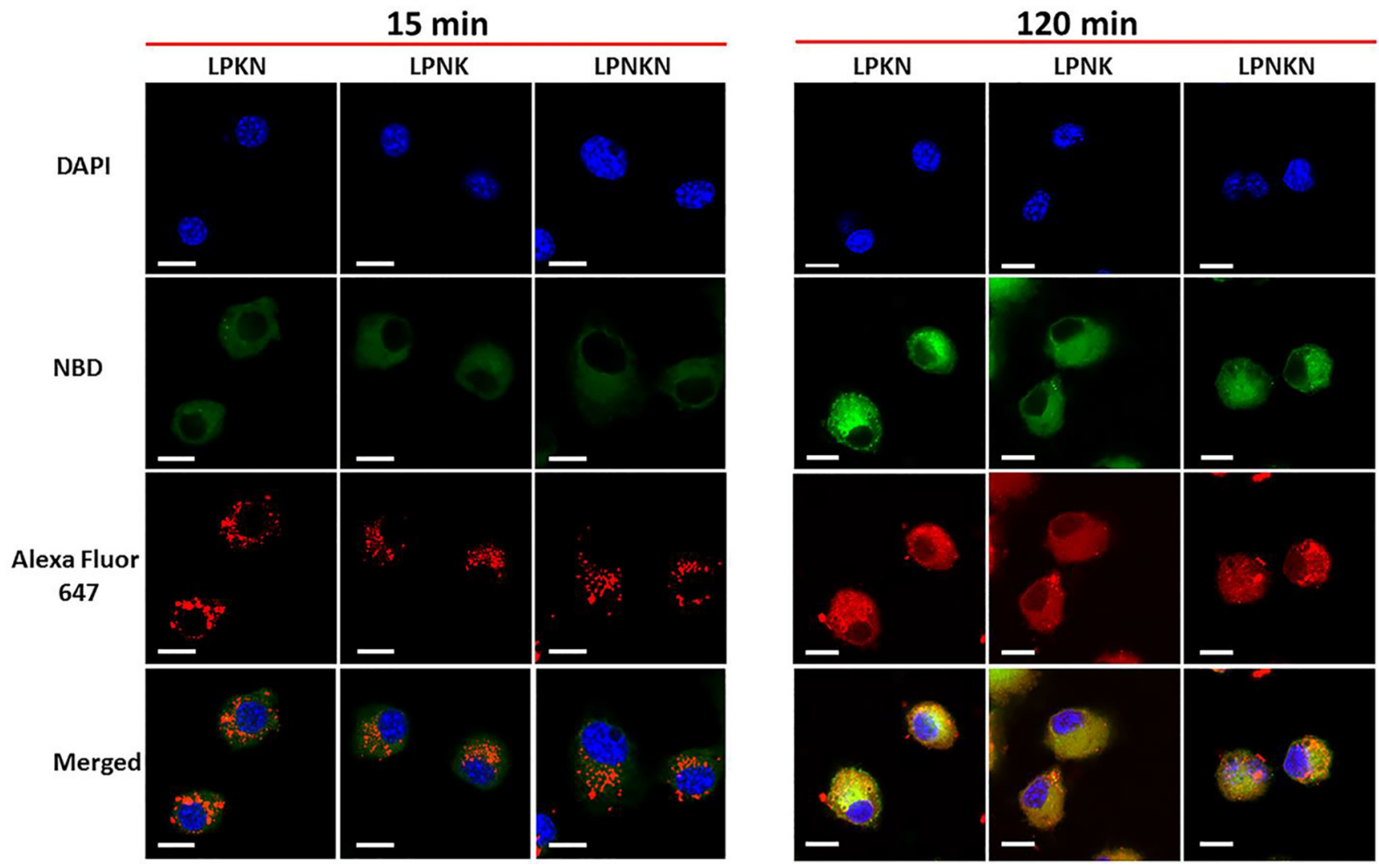

Fig. 4. Uptake of nanovaccine NPs by dendritic cells analyzed by CLSM. The lipid-layer of hybrid NPs was labeled by NBD. Nic-hapten on KLH was substituted with AF647 to provide fluorescence. Cells were treated with $20 \mu \mathrm{g}$ of nanovaccine NPs for $15 \mathrm{~min}$ or $120 \mathrm{~min}$. Scale bars represent $10 \mu \mathrm{m}$.

A

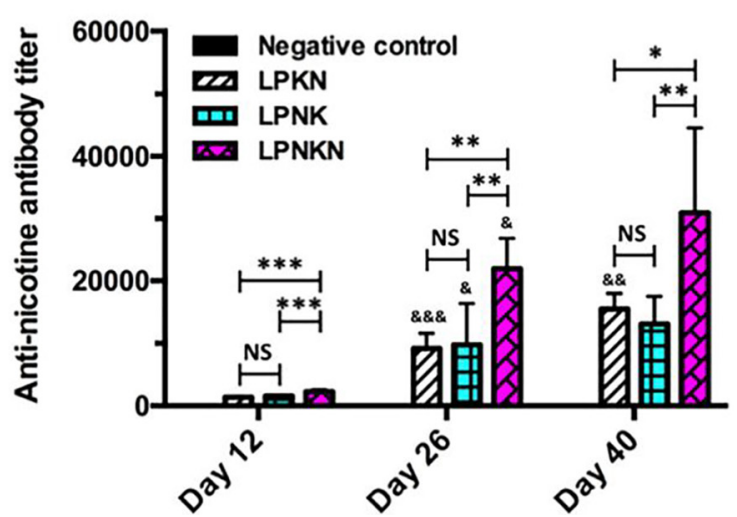

B

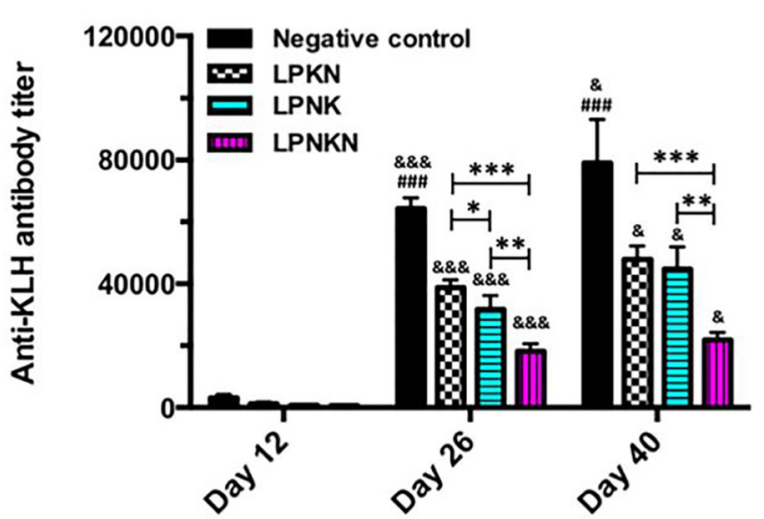

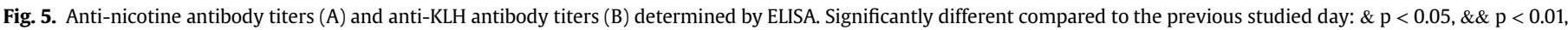

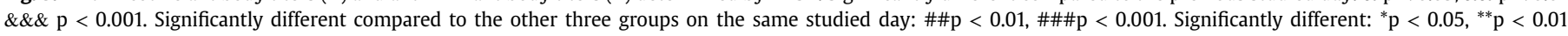
*** $\mathrm{p}<0.001$.

LPNKN induced higher titers of all four IgG subtypes over LPNK and LPNKN, especially for IgG1 and IgG2a. Interestingly, although the total IgG titers of LPKN and LPNK were very close (Fig. 5A), LPNK generated significantly higher levels of IgG2a than LPKN. The relative magnitude of Th1 versus Th2 immune response induced by nanovaccines was assessed by the Th1/Th2 index. The Th1/Th2 indexes for LPKN, LPNK, and LPNKN were $0.043 \pm 0.042$, $0.430 \pm 0.288$, and $0.191 \pm 0.136$, respectively, which were all significantly less than 1 . The Th1/Th2 index data indicates that the immune responses induced by the nanovaccines, regardless of hapten localizations, was Th2-skewed (humoral response dominated). Interestingly, LPNKN and LPNK resulted in a more balanced
Th1-Th2 response than LPKN.

\subsection{Pharmacokinetic efficacy of nanovaccines}

Pharmacokinetic efficacy of nanovaccines with different hapten localizations was tested in mice. Mice received a dose of $0.06 \mathrm{mg} / \mathrm{kg}$ nicotine on day 42 , and the nicotine levels in the serum and brain 3 min post-administration were analyzed. Serum nicotine levels were shown in Fig. 8A. The blank group had a serum nicotine level of $12.5 \mathrm{ng} / \mathrm{mL}$. Compared to the blank group, the nicotine levels in mice injected with LPKN, LPNK or LPNKN increased by $79.2 \%, 20 \%$, and $192.0 \%$, respectively. These data indicate that LPNKN had the 
A

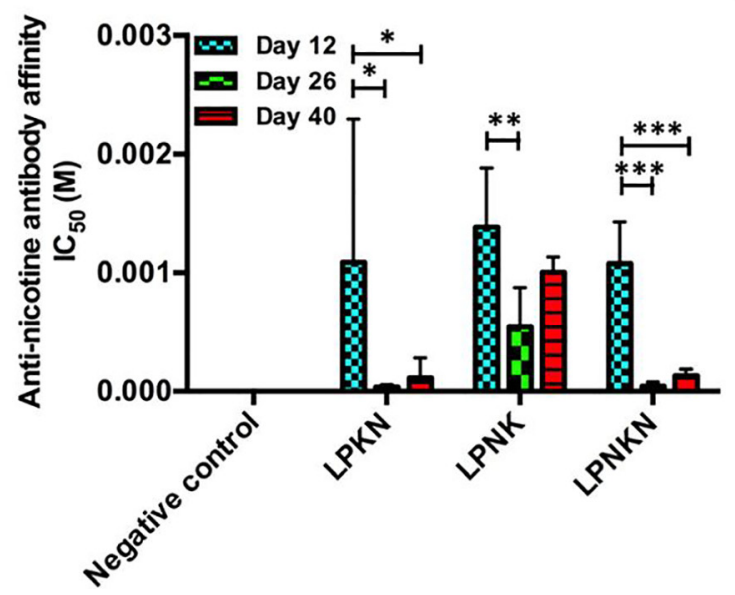

B

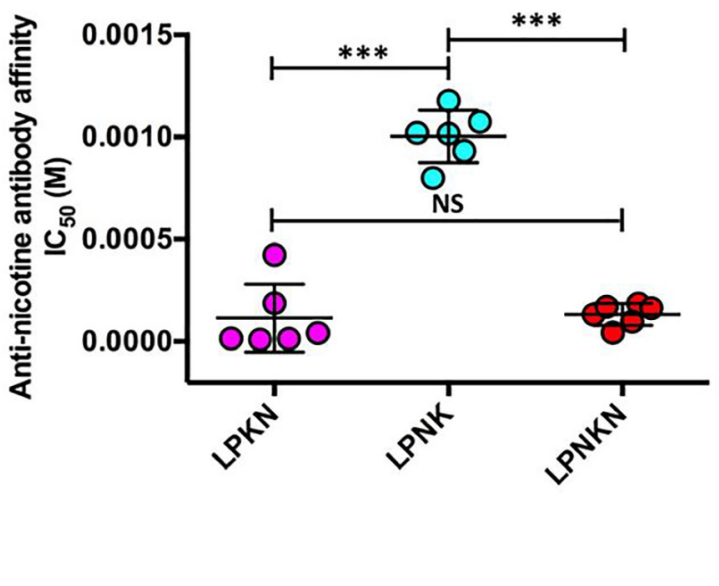

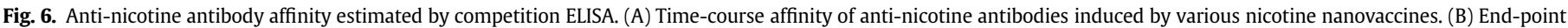
comparison of antibody's affinity among different hapten localization nanovaccine groups on day 40 . Significantly different: ${ }^{*} \mathrm{p}<0.05$, ${ }^{* *} \mathrm{p}<0.01$, ${ }^{* * *} \mathrm{p}<0.001$.

best ability to retain nicotine in serum. Nicotine levels in the brain were shown in Fig. 8B. The brain nicotine level in the blank group was $98.8 \mathrm{ng} / \mathrm{g}$. The percent reductions in brain nicotine levels were 49.4\%, $41.3 \%$, and $66.9 \%$ for LPKN, LPNK, and LPNKN vaccinated groups, respectively. Based on the above results, LPNKN has the best ability of blocking nicotine from entering the brain.

\subsection{Safety of nanovaccines}

The safety of nanovaccines was evaluated histopathologically (Fig. 9). Major organs of mice, including heart, kidney, lung, liver, and spleen, were stained with $\mathrm{H} \& \mathrm{E}$ and examined. No significant differences were detected between the blank (PBS) and the three nanovaccine groups, in all examined organs. Moreover, no detectable difference was observed among the nanovaccines with different hapten localizations. Also, the behavioral and physical conditions of mice during the entire period of study were monitored. No short-term effects, including local site reaction, elevation in body temperature, apparent abnormal behavior, and abnormal food and water consumption, were detected in all groups. Thus, the nanovaccines, regardless of hapten localization, are considered safe.

\section{Discussion}

Nicotine vaccines are a promising strategy for future treatment against nicotine addiction. Currently, conjugate vaccines are the prevalent and most-studied nicotine vaccines. However, their intrinsic shortcomings, such as low nicotine loading capacity, low bioavailability, poor recognition and uptake by immune cells, and difficulty in incorporation of adjuvants, limit their immunological efficacy $[9,25]$. Nanoparticles have been widely studied for delivery of drugs and vaccines [36-40]. Nanoparticles are able to maintain
A
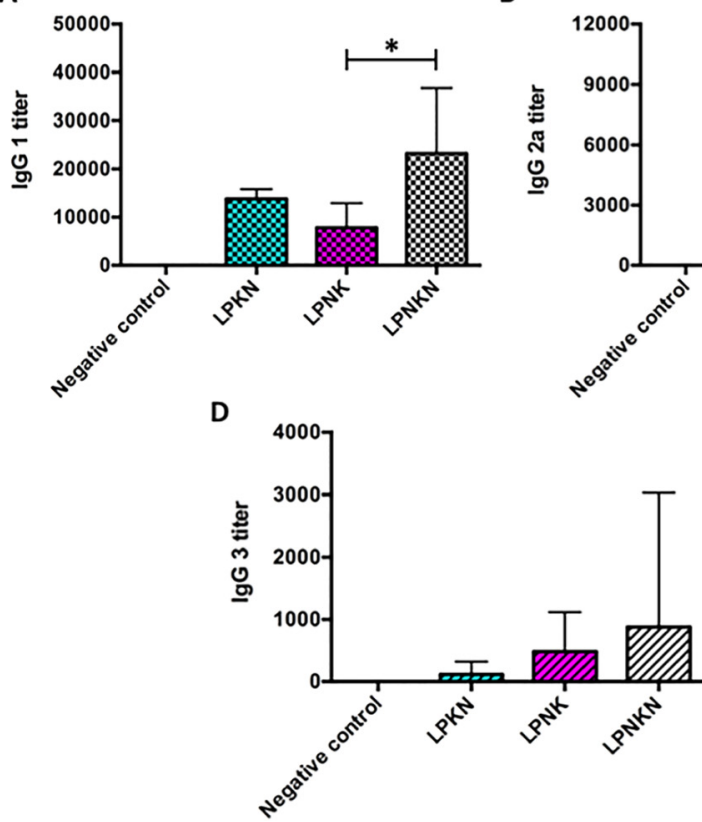

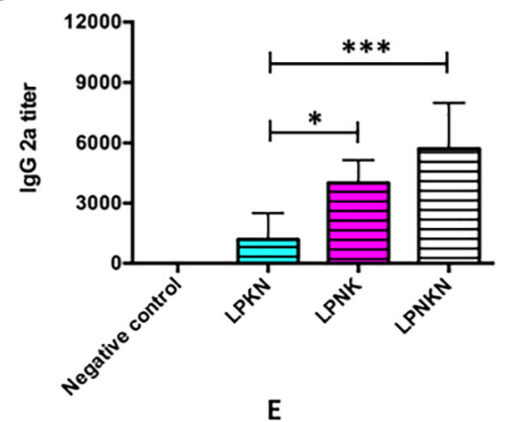

$\mathbf{E}$

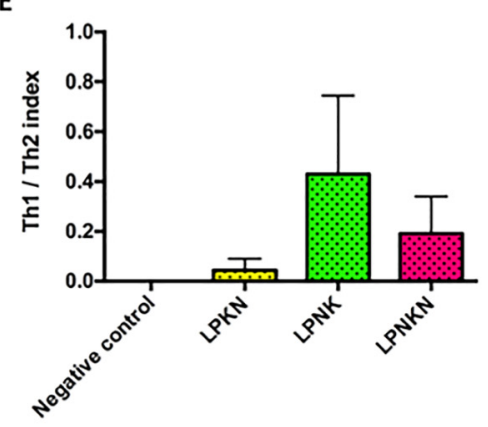

C

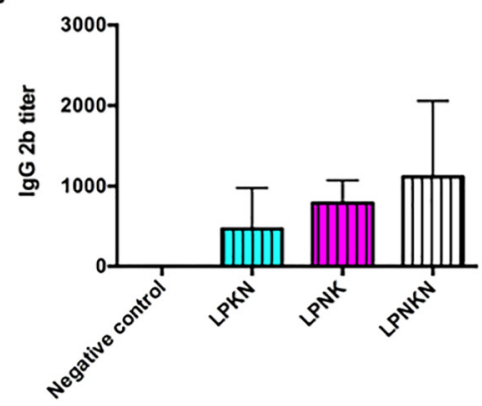

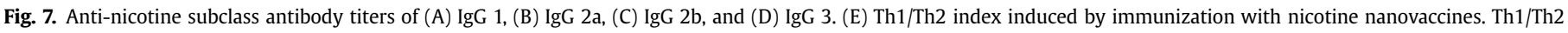
index $=(\operatorname{IgG} 2 \mathrm{a}+\operatorname{IgG3}) / 2 / \operatorname{IgG} 1$. Significantly different: ${ }^{*} \mathrm{p}<0.05,{ }^{* * *} \mathrm{p}<0.001$. 
A

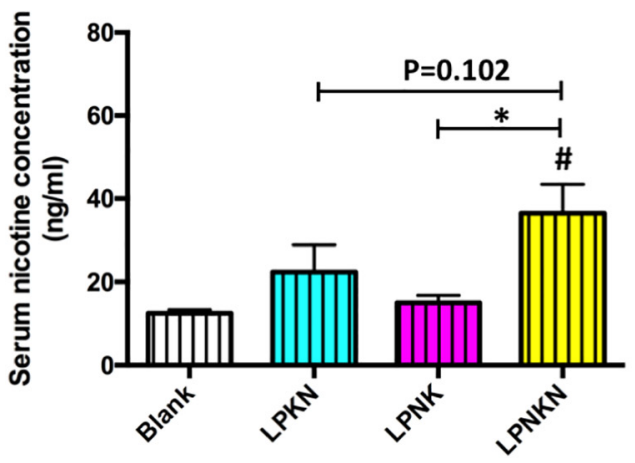

B

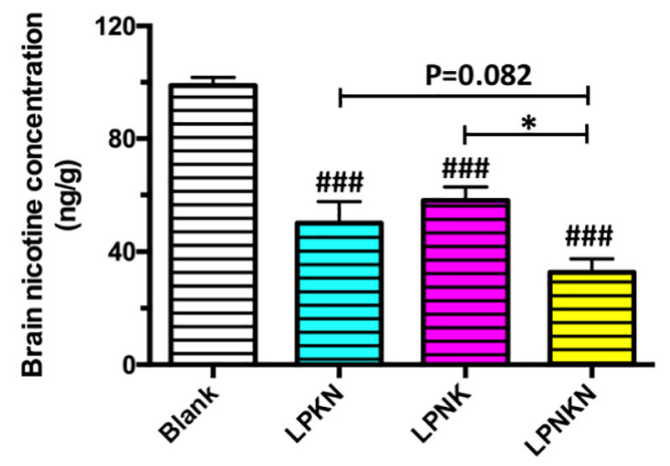

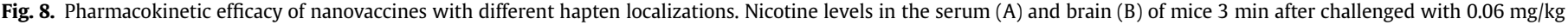
nicotine. Data were reported as means \pm standard error. Significantly different compared to the blank group: \#p $<0.05$, \#\#\#p $<0.001$. Significantly different: ${ }^{*} \mathrm{p}<0.05$.

the activity of payloads and enhance delivery efficiency. In addition, high payload loading capacity, improved bioavailability, and controlled payload release can be achieved by nanoparticles [41-44]. These advantages inspired us to develop the nextgeneration nanoparticle-based nicotine vaccines to circumvent the innate shortfalls of conjugate nicotine vaccines. In our previous work, we conceptualized a lipid-polymeric hybrid nanoparticlebased nicotine nanovaccine, and demonstrated that its immunogenicity was significantly higher than that of the conjugate vaccine [26]. In this study, we rationalized the nanovaccine design by investigating the impact of hapten localization on its immunogenicity and efficacy. The nanovaccines developed in this current study differ in hapten localization, but have similar overall hapten loading, average size and size distribution, and surface charge (Table 1).

The nicotine nanovaccines are based on a lipid-polymeric hybrid nanoparticle. TEM images suggested the successful formation of a core-shell hybrid structure (Fig. 2A). In this study, each component of the hybrid nanoparticles was adopted for specific purposes. PLGA core not only supplies extra rigidity to liposomes to stabilize the nanoparticle system [45], but also controls the size of nanovaccines. We previously demonstrated that nicotine nanovaccines of $100 \mathrm{~nm}$ had a significantly higher immunogenicity than that of $500 \mathrm{~nm}$ [26]. In this study, PLGA core was fabricated by a nanoprecipitation method [46] so as to result in a precisely controlled particle size. Additionally, PLGA core would provide a cargo space for encapsulation and controlled release of molecular adjuvants [47,48]. The lipid shell was composed of DOTAP, cholesterol, DSPE-PEG2000maleimide, and DSPE-PEG2000-amine. DOTAP [49], a cationic lipid, produces a positively-charged nanoparticle surface (Table 1) and may promote the interaction between nanovaccines and negatively-charged immune cells, thus leading to enhanced internalization of vaccine particles. Cholesterol acts as a stabilizer in the

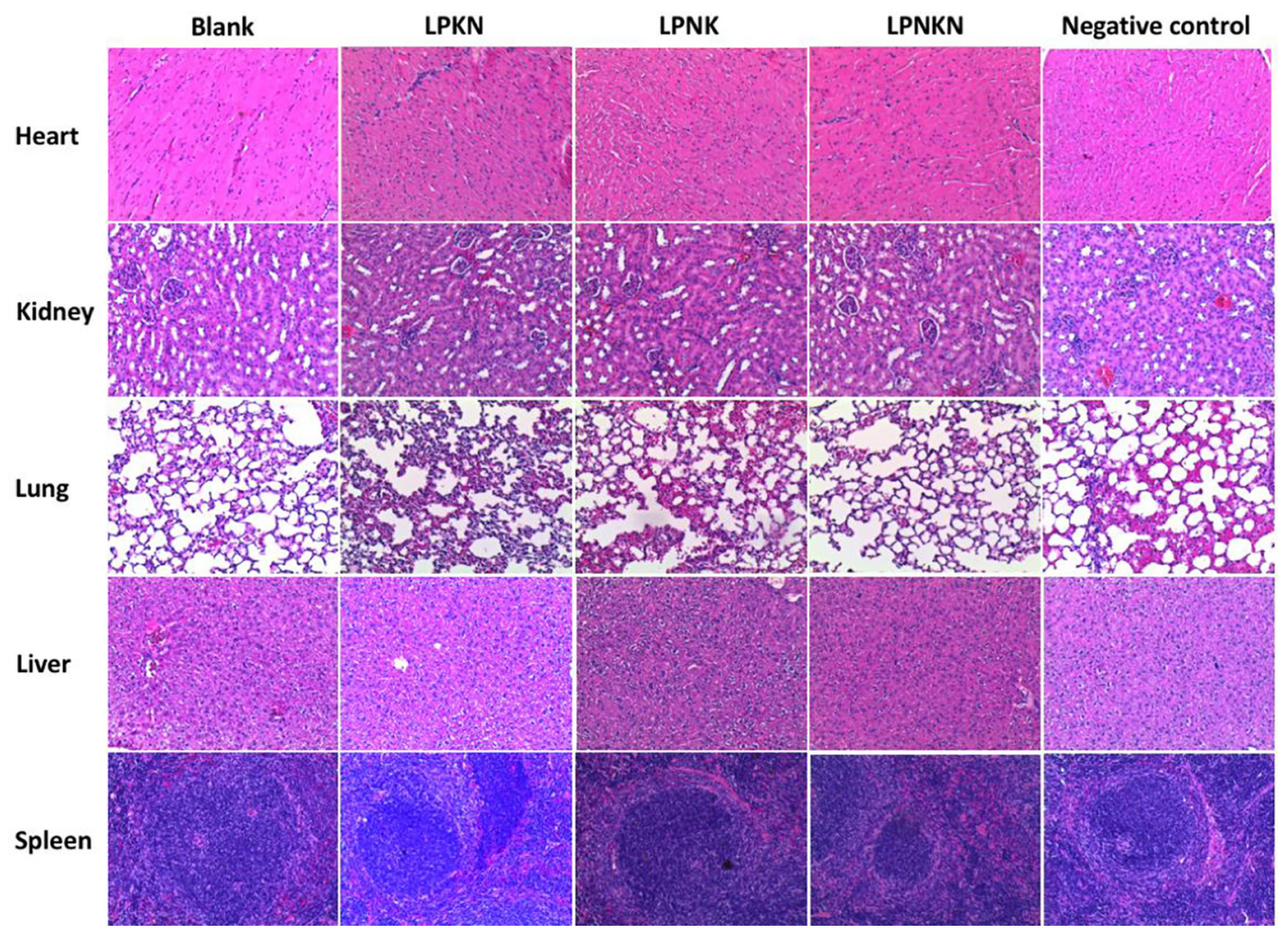

Fig. 9. H\&E staining of the sections of major organs including heart, kidney, lung, liver, and spleen harvested from the mice immunized with different nicotine vaccines. 
lipid layer to improve the stability of nanovaccines [50]. DSPEPEG2000-maleimide allows conjugation of multiple KLH/Nic-KLH to nanoparticle surface, leading to a high loading efficiency of protein antigen. DSPE-PEG2000-amine enables conjugation of Nichapten onto nanoparticle surface. Additionally, PEGylation may prolong the circulation time of nanovaccines and increase the bioavailability of vaccines [51].

To induce an immune response, vaccine particles need to be efficiently internalized and processed by antigen presenting cells (APCs) [9]. Both the FCA and CLSM data suggested that the nanovaccines, regardless of hapten localization, were taken up by DCs efficiently (Figs. 3 and 4), possibly due to the positively charged surface of nanovaccines. Specifically, more than $75 \%$ of the cells had taken up nanoparticles within 120 min. The efficient internalization of nanovaccines may provide sufficient antigens to induce a quick development of immune response. Moreover, CLSM images indicated the sufficient processing of protein antigens to peptide antigens within $120 \mathrm{~min}$. Efficient processing of protein antigens may ensure effective activation of T-helper cells, making the immune response fervent and long-lasting. Interestingly, the uptake of LPKN particles is slightly more efficient than that of LPNK and LPNKN particles. This may be caused by the high PEG-grafting in LPNK and LPNKN, as it has been reported that PEG-grafting might decrease the uptake of nanoparticles [52].

Previous studies suggested that anti-nicotine antibody levels largely determine the smoking cessation efficacy $[16,17]$. LPNKN induced significantly higher anti-nicotine antibody titers than LPKN and LPNK, while the antibody levels of LPKN and LPNK were comparable (Fig. 5A). This finding suggests that the immunogenicity of nanovaccines could be improved by conjugating Nichaptens onto both carrier protein and nanoparticle surface, instead of onto only one. Anti-KLH antibody is considered a nonspecific byproduct. Production of anti-KLH antibody would cause wastage of vaccines to generate antibodies against KLH rather than nicotine. Meanwhile, high levels of anti-KLH antibody may neutralize nanovaccines injected during booster immunizations. Interestingly, LPNKN induced the lowest anti-KLH antibody titers among the three nanovaccines with different hapten localizations. This may be explained by the following facts. First, conjugation of Nic-hapten to KLH masked sufficient amounts of immunogenic epitopes on KLH. Second, the shielding effect of PEG-grafting [53] on LPNKN might decrease the recognition of immunogenic epitopes on KLH by immune cells.

Based on the ELISA results (Fig. 5), LPNKN had the highest immunogenicity among the three nanovaccines, eliciting the highest titers of anti-nicotine antibody and the lowest levels of anti-KLH antibody. Although we do not have direct evidences to show the mechanism, the following may explain the immunogenic differences among the nanovaccines that had similar overall hapten densities but different hapten localizations. Generation of an effective humoral immune response against nicotine requires two pivotal processes: the first process is activation of nicotine-specific $\mathrm{B}$ cells; the second process is generation of T-helper cells and the interaction between T-helper cells and B cells, which are required for the maturation of nicotine-specific B cells to antibody-secreting cells [9]. Generation of T-helper cells is realized by specific recognition of T cell epitopes (displayed on APCs) by T cells. Maturation of nicotine-specific $B$ cells is facilitated by specific recognition of T cell epitopes (displayed on B cells) by T helper cells [9]. As for LPKN, the process of B cell activation was sufficient. However, Nic-haptens were localized on KLH at a high density. This might mask some of the $\mathrm{T}$ cell epitopes, and thus causing the $\mathrm{B}$ cell maturation to be insufficient. For LPNKN, B cell activation was efficient. As less Nichaptens were localized on $\mathrm{KLH}, \mathrm{T}$ cell epitopes were minimally masked. Thus, the B cell maturation process was also sufficient. As for LPNK, no Nic-haptens were conjugated to KLH, and thus the immunogenic epitopes of KLH were fully exposed. Therefore, a portion of nanovaccines would be used to generate antibodies against KLH rather than nicotine, leading to a low immunogenicity against nicotine. However, cellular mechanistic study is not within the scope of this work. The proposed mechanism needs to be verified in the future.

Anti-nicotine antibody's affinity is a critical factor influencing the efficacy of a nicotine vaccine [9]. Interestingly, antibodies induced by LPKN and LPNKN had significantly higher affinity than that by LPNK (Fig. 6). This indicates that hapten localization appeared to affect anti-nicotine antibody's affinity. Analysis of subtype distribution of anti-nicotine IgG antibody revealed that LPNK generated higher percentages of IgG2a, IgG2b, and IgG3 than LPKN, especially IgG2a (Fig. 7), although their total IgG titers were close (Fig. 5). Hapten localization thus influences the anti-nicotine IgG subtype distribution, and tends to induce widely-distributed IgG subtypes. Th1/Th2 index is an indicator of the polarization of immune responses [54]. Although LPNK and LPNKN induced more balanced Th1-Th2 immune responses than LPKN (Fig. 7E), all three nanovaccines induced Th2-skewed humoral responses (Th1/Th2 significantly less than 1). Th2-skewed immune responses are considered desirable for nicotine vaccine design, as the efficacy of reducing the rewarding effects of nicotine is dependent on the magnitude of the humoral response. Consistent with the immunogenicity and affinity data, LPNKN resulted in a better pharmacokinetic (PK) efficacy of retaining nicotine in serum and blocking nicotine from entering the brain than LPKN and LPNK (Fig. 8). The PK study results suggest the efficacy of nicotine nanovaccines could be improved by conjugating Nic-haptens to both stimulating protein and nanoparticle surface. In our previous study, we found that LPKN induced an anti-nicotine antibody titer of $\sim 9,000$ on day 41 and resulted in a $32 \%$ brain nicotine reduction [26]. However, in this study, we found that LPKN elicited an antibody titer of $\sim 15,000$ on day 40 and blocked $49 \%$ of nicotine from entering the brain of mice. This difference can be attributed to that LPKN prepared in the two studies differed in Nic-KLH conjugation capacity (the mass ratio of conjugated Nic-KLH to hybrid nanoparticles). Future work needs to be done to establish an optimal Nic-carrier protein conjugation capacity. In addition, we previously demonstrated that LPKN induced a significantly better pharmacokinetic efficacy than the conjugate vaccine. The finding in this current study may further improve the efficacy of hybrid nanoparticle-based nicotine nanovaccines. It should be pointed out that no molecular adjuvants were incorporated to the nanovaccines in this study. A study is undergoing to further improve the immunogenicity of nanovaccines by selecting potent molecular adjuvants.

In summary, a lipid-polymeric hybrid nanoparticle-based nextgeneration nicotine nanovaccine was successfully fabricated and tested with a focus on studying the impact of hapten localization on its immunogenicity and efficacy. Results from mice trials suggested that vaccines with hapten molecules conjugated on both the carrier protein and nanoparticle surface have better immunogenicity and pharmacokinetic efficacy against nicotine than vaccines with haptens only on protein or nanoparticle surface. The findings of this study provide a novel strategy to improve the immunogenicity and efficacy of the next-generation nanoparticle-based nicotine vaccine, and can be applied to the design of other nanoparticle-based vaccines. Based on all the reported results, hybrid nanoparticlebased next-generation nicotine nanovaccines hold great promise as future clinical vaccines against nicotine addiction.

\section{Conflict of interest}

The authors declare no competing financial interests. 


\section{Acknowledgment}

This work was financially supported by National Institute on Drug Abuse (U01DA036850). We thank Jie Zhu for his help on collecting FT-IR data.

\section{References}

[1] Tobacco, Fact Sheet Number 339, World Health Organization, 2015.

[2] N.L. Benowitz, Nicotine addiction, N. Engl. J. Med. 362 (2010) 2295-2303.

[3] J.J. Prochaska, N.L. Benowitz, The past, present, and future of nicotine addiction therapy, Annu. Rev. Med. 67 (2016) 467-486.

[4] J.R. Hughes, J. Keely, S. Naud, Shape of the relapse curve and long-term abstinence among untreated smokers, Addiction 99 (2004) 29-38.

[5] M. Paolini, M. De Biasi, Mechanistic insights into nicotine withdrawal, Biochem. Pharmacol. 82 (2011) 996-1007.

[6] L.F. Stead, R. Perera, C. Bullen, D. Mant, J. Hartmann-Boyce, K. Cahill, et al., Nicotine replacement therapy for smoking cessation, Cochrane Db Syst. Rev, (11) (2012). Article No. is CD000146.

[7] M.E. Piper, E.B. Federman, D.E. McCarthy, D.M. Bolt, S.S. Smith, M.C. Fiore, et al., Efficacy of bupropion alone and in combination with nicotine gum, Nicotine Tob. Res. 9 (2007) 947-954.

[8] C.F.N. Koegelenberg, F. Noor, E.D. Bateman, R.N. van Zyl-Smit, A. Bruning J.A. O'Brien, et al., Efficacy of varenicline combined with nicotine replacement therapy vs varenicline alone for smoking cessation a randomized clinical trial, Jama-J Am. Med. Assoc. 312 (2014) 155-161.

[9] P.R. Pentel, M.G. LeSage, New directions in nicotine vaccine design and use, Adv. Pharmacol. 69 (2014) 553-580.

[10] T. Raupach, P.H. Hoogsteder, C.P. Onno van Schayck, Nicotine vaccines to assist with smoking cessation: current status of research, Drugs 72 (2012) e1-16.

[11] M.L. Goniewicz, M. Delijewski, Nicotine vaccines to treat tobacco dependence, Hum. Vacc Immunother. 9 (2013) 13-25.

[12] D.E. Keyler, S.A. Roiko, C.A. Earley, M.P. Murtaugh, P.R. Pentel, Enhanced immunogenicity of a bivalent nicotine vaccine, Int. Immunopharmacol. 8 (2008) 1589-1594.

[13] M.J. McCluskie, J. Thorn, P.R. Mehelic, P. Kolhe, K. Bhattacharya, J.I. Finneman, et al., Molecular attributes of conjugate antigen influence function of antibodies induced by anti-nicotine vaccine in mice and non-human primates, Int. Immunopharmacol. 25 (2015) 518-527.

[14] K.D. Miller, R. Roque, C.H. Clegg, Novel anti-nicotine vaccine using a trimeric coiled-coil hapten carrier, Plos One (2014) 9.

[15] M. De Blasi, I. McLaughlin, E.E. Perez, P.A. Crooks, L.P. Dwoskin, M.T. Bardo, et al., Scientific Overview: 2013 BBC Plenary Symposium on Tobacco Addiction (Vol. 141, Pg 107, 2014). Drug Alcohol Depen, 2014, 144:290-.

[16] D.K. Hatsukami, D.E. Jorenby, D. Gonzales, N.A. Rigotti, E.D. Glover, C.A. Oncken, et al., Immunogenicity and smoking-cessation outcomes for a novel nicotine immunotherapeutic, Clin. Pharmacol. Ther. 89 (2011) 392-399.

[17] J. Cornuz, S. Zwahlen, W.F. Jungi, J. Osterwalder, K. Klingler, G. van Melle, et al., A vaccine against nicotine for smoking cessation: a randomized controlled trial, Plos One (2008) 3.

[18] D.C. Pryde, L.H. Jones, D.P. Gervais, D.R. Stead, D.C. Blakemore, M.D. Selby, et al., Selection of a novel anti-nicotine vaccine: influence of antigen design on antibody function in mice, Plos One (2013) 8.

[19] S.H.L. de Villiers, N. Lindblom, G. Kalayanov, S. Gordon, I. Baraznenok, A. Malmerfelt, et al., Nicotine hapten structure, antibody selectivity and effect relationships: results from a nicotine vaccine screening procedure, Vaccine 28 (2010) 2161-2168.

[20] X.Y. Chen, M. Pravetoni, B. Bhayana, P.R. Pentel, M.X. Wu, High immunogenicity of nicotine vaccines obtained by intradermal delivery with safe adjuvants, Vaccine 31 (2012) 159-164.

[21] M.J. McCluskie, D.C. Pryde, D.P. Gervais, D.R. Stead, N.L. Zhang, M. Benoit, et al., Enhancing immunogenicity of a 3' aminomethylnicotine-DT-conjugate antinicotine vaccine with $\mathrm{CpG}$ adjuvant in mice and non-human primates, Int. Immunopharmacol. 16 (2013) 50-56.

[22] J.W. Lockner, J.M. Lively, K.C. Collins, J.C.M. Vendruscolo, M.R. Azar, K.D. Janda, A conjugate vaccine using enantiopure hapten imparts superior nicotinebinding capacity, J. Med. Chem. 58 (2015) 1005-1011.

[23] K.C. Collins, K.D. Janda, Investigating hapten clustering as a strategy to enhance vaccines against drugs of Abuse, Bioconjug Chem. 25 (2014) 593-600.

[24] Y. Hu, H. Zheng, W. Huang, C.M. Zhang, A novel and efficient nicotine vaccine using nano-lipoplex as a delivery vehicle, Hum. Vacc Immunother. 10 (2014) $64-72$.

[25] H. Zheng, Y. Hu, W. Huang, S. de Villiers, P. Pentel, J.F. Zhang, et al., Negatively charged carbon nanohorn supported cationic liposome nanoparticles: a novel delivery vehicle for anti-nicotine vaccine, J. Biomed. Nanotechnol. 11 (2015) 2197-2210.

[26] Z. Zhao, Y. Hu, R. Hoerle, M. Devine, M. Raleigh, P. Pentel, et al., A nanoparticle-based nicotine vaccine and the influence of particle size on its immunogenicity and efficacy, Nanomed-Nanotechnol 13 (2016) 443-454.

[27] Z. Zhao, K. Powers, Y. Hu, M. Raleigh, P. Pentel, C. Zhang, Engineering of a hybrid nanoparticle-based nicotine nanovaccine as a next-generation immunotherapeutic strategy against nicotine addiction: a focus on hapten density, Biomaterials 123 (2017) 107-117.

[28] N.T. Jacob, J.W. Lockner, J.E. Schlosburg, B.A. Ellis, L.M. Eubanks, K.D. Janda, Investigations of enantiopure nicotine haptens using an adjuvanting carrier in anti-nicotine vaccine development, J. Med. Chem. 59 (2016) 2523-2529.

[29] J. Parra, A. Abad-Somovilla, J.V. Mercader, T.A. Taton, A. Abad-Fuentes, Carbon nanotube-protein carriers enhance size-dependent self-adjuvant antibody response to haptens, J. Control Release 170 (2013) 242-251.

[30] B.R. Sloat, M.A. Sandoval, A.M. Hau, Y. He, Z. Cui, Strong antibody responses induced by protein antigens conjugated onto the surface of lecithin-based nanoparticles, J. Control Release 141 (2010) 93-100.

[31] H. Babahosseini, V. Srinivasaraghavan, Z.M. Zhao, F. Gillam, E. Childress, J.S. Strobl, et al., The impact of sphingosine kinase inhibitor-loaded nanoparticles on bioelectrical and biomechanical properties of cancer cells, Lab. Chip 16 (2016) 188-198.

[32] Y. Hu, Z.M. Zhao, M. Ehrich, K. Fuhrman, C.M. Zhang, In vitro controlled release of antigen in dendritic cells using pH-sensitive liposome-polymeric hybrid nanoparticles, Polymer 80 (2015) 171-179.

[33] R. Jalah, O.B. Torres, A.V. Mayorov, F. Li, J.F. Antoline, A.E. Jacobson, et al., Efficacy, but not antibody titer or affinity, of a heroin hapten conjugate vaccine correlates with increasing hapten densities on tetanus toxoid, but not on CRM197 carriers, Bioconjug Chem. 26 (2015) 1041-1053.

[34] M. Pravetoni, D.E. Keyler, R.R. Pidaparthi, F.I. Carroll, S.P. Runyon, M.P. Murtaugh, et al., Structurally distinct nicotine immunogens elicit antibodies with nonoverlapping specificities, Biochem. Pharmacol. 83 (2012) 543-550.

[35] S.H.L. de Villiers, K.E. Cornish, A.J. Troska, M. Pravetoni, P.R. Pentel, Increased efficacy of a trivalent nicotine vaccine compared to a dose-matched monovalent vaccine when formulated with alum, Vaccine 31 (2013) 6185-6193.

36] S. Thangavel, T. Yoshitomi, M.K. Sakharkar, Y. Nagasaki, Redox nanoparticle increases the chemotherapeutic efficiency of pioglitazone and suppresses its toxic side effects, Biomaterials 99 (2016) 109-123.

[37] J. Liu, T. Wei, J. Zhao, Y.Y. Huang, H. Deng, A. Kumar, et al., Multifunctional aptamer-based nanoparticles for targeted drug delivery to circumvent cancer resistance, Biomaterials 91 (2016) 44-56.

[38] Y. Qian, H.L. Jin, S. Qiao, Y.F. Dai, C. Huang, L.S. Lu, et al., Targeting dendritic cells in lymph node with an antigen peptide-based nanovaccine for cancer immunotherapy, Biomaterials 98 (2016) 171-183.

[39] S.Y. Yan, B.E. Rolfe, B. Zhang, Y.H. Mohammed, W.Y. Gu, Z.P. Xu, Polarized immune responses modulated by layered double hydroxides nanoparticle conjugated with CpG, Biomaterials 35 (2014) 9508-9516.

[40] R.A. Rosalia, L.J. Cruz, S. van Duikeren, A.T. Tromp, A.L. Silva, W. Jiskoot, et al. CD40-targeted dendritic cell delivery of PLGA-nanoparticle vaccines induce potent anti-tumor responses, Biomaterials 40 (2015) 88-97.

[41] M.C. Chen, K. Sonaje, K.J. Chen, H.W. Sung, A review of the prospects for polymeric nanoparticle platforms in oral insulin delivery, Biomaterials 32 (2011) 9826-9838.

[42] B. Mandal, H. Bhattacharjee, N. Mittal, H. Sah, P. Balabathula, L.A. Thoma, et al, Core-shell-type lipid-polymer hybrid nanoparticles as a drug delivery platform, Nanomed-Nanotechnol 9 (2013) 474-491.

43] S.R. Grobmyer, G.Y. Zhou, L.G. Gutwein, N. Iwakuma, P. Sharma, S.N. Hochwald, Nanoparticle delivery for metastatic breast cancer, NanomedNanotechnol 8 (2012) S21-S30.

[44] K. Park, Controlled drug delivery systems: past forward and future back, J. Control Release 190 (2014) 3-8.

[45] L.F. Zhang, S. Granick, How to stabilize phospholipid liposomes (using nanoparticles), Nano Lett. 6 (2006) 694-698.

46] P.F. Zhao, M.B. Zheng, C.X. Yue, Z.Y. Luo, P. Gong, G.H. Gao, et al., Improving drug accumulation and photothermal efficacy in tumor depending on size of ICG loaded lipid-polymer nanoparticles, Biomaterials 35 (2014) 6037-6046.

[47] M. Mueller, W. Reichardt, J. Koerner, M. Groettrup, Coencapsulation of tumor lysate and CpG-ODN in PLGA-microspheres enables successful immunotherapy of prostate carcinoma in TRAMP mice, J. Control Release 162 (2012) 159-166.

48] O. Wang, M.T. Tan, B.P. Keegan, M.A. Barry, M.J. Heffernan, Time course study of the antigen-specific immune response to a PLGA microparticle vaccine formulation, Biomaterials 35 (2014) 8385-8393.

[49] K.Y. Shen, H.Y. Liu, H.J. Li, C.C. Wu, G.G. Liou, Y.C. Chang, et al., A novel liposomal recombinant lipoimmunogen enhances anti-tumor immunity, J. Control Release 233 (2016) 57-63.

[50] T. Nakazawa, S. Nagatsuka, O. Yukawa, Effects of membrane stabilizing agents and radiation on liposomal membranes, Drug Exp. Clin. Res. 12 (1986) 831-835.

[51] A. Ibricevic, S.P. Guntsen, K. Zhang, R. Shrestha, Y.J. Liu, J.Y. Sun, et al. PEGylation of cationic, shell-crosslinked-knedel-like nanoparticles modulates inflammation and enhances cellular uptake in the lung, Nanomed-Nanotechnol 9 (2013) 912-922.

[52] B. Pelaz, P. del Pino, P. Maffre, R. Hartmann, M. Gallego, S. Rivera-Fernandez, et al., Surface functionalization of nanoparticles with polyethylene glycol: effects on protein adsorption and cellular uptake, Acs Nano 9 (2015) 6996-7008.

[53] F.M. Mickler, Y. Vachutinsky, M. Oba, K. Miyata, N. Nishiyama, K. Kataoka, et al., Effect of integrin targeting and PEG shielding on polyplex micelle internalization studied by live-cell imaging, J. Control Release 156 (2011) 364-373.

[54] M. Moser, K.M. Murphy, Dendritic cell regulation of TH1-TH2 development, Nat. Immunol. 1 (2000) 199-205. 